EPJ Web of Conferences 66, 02012 (2014)

DOI: $10.1051 /$ epjconf/ 20146602012

(C) Owned by the authors, published by EDP Sciences, 2014

\title{
Quadrupole collectivity in neutron-rich Cd isotopes
}

S. Bönig ${ }^{1, a}$, S. llieva ${ }^{1}$, T. Kröll ${ }^{1}$, M. Scheck ${ }^{1}$, D. Balabanski², C. Bauer ${ }^{1}$, A. Blazhev ${ }^{3}$, T. Bloch ${ }^{1}$, D. Deleanu ${ }^{4}$, J. Diriken ${ }^{5,6}$, P. Fernier ${ }^{7}$, R. Gernhäuser ${ }^{8}$, K. Hadyńska-Klęk ${ }^{9}$, A. Jungclaus ${ }^{10}$, R. Lutter ${ }^{8,11}$ A. Negret ${ }^{4}$, K. Nowak ${ }^{8}$, R. Orlandi ${ }^{10}$, J. Pakarinen ${ }^{7}$, G. Rainovski ${ }^{12}$, T.R. Rodríguez ${ }^{1}$, M. von Schmid ${ }^{1}$, M. Seidlitz ${ }^{3}$, B. Siebeck ${ }^{3}$, G.S. Simpson ${ }^{13}$, A. Illana Sisón ${ }^{10}$, R. Stegmann ${ }^{1}$, T. Stora ${ }^{7}$, P.G. Thirolf ${ }^{11}$, M. Thürauf ${ }^{1}$, M.J. Vermeulen ${ }^{14}$, D. Voulot ${ }^{7}$, N. Warr' ${ }^{3}$, F. Wenander ${ }^{7}$, H. De Witte ${ }^{5}$, and MINIBALL Collaboration

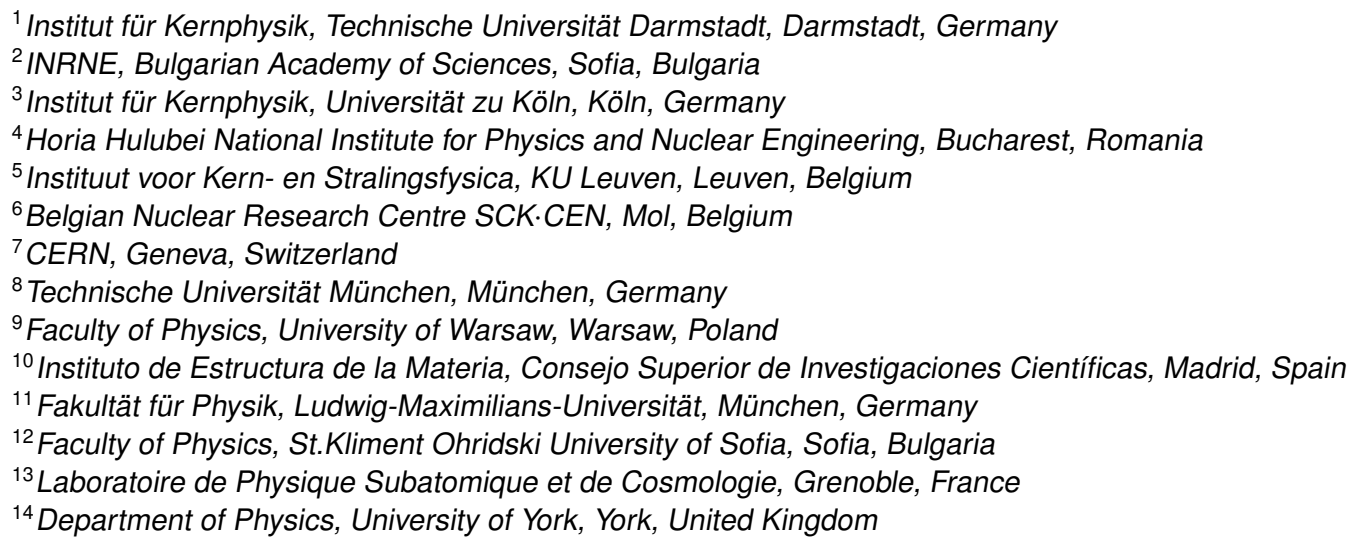

\begin{abstract}
The investigation of the excitation energies of the $2_{1}^{+}$-states in the neutron-rich $\mathrm{Cd}$ isotopes shows an irregular behaviour when approaching the neutron shell-closure at $\mathrm{N}=82$. The energy of the $2_{1}^{+}$-state in ${ }^{128} \mathrm{Cd}$ is lower than the one in ${ }^{126} \mathrm{Cd}$. The transition strength $\mathrm{B}\left(\mathrm{E} 2,0_{\mathrm{gs}}^{+} \rightarrow 2_{1}^{+}\right)$in the even isotopes ${ }^{122-128} \mathrm{Cd}$ was measured in Coulomb excitation experiments with the high-purity germanium detector array MINIBALL at REXISOLDE (CERN). The values for ${ }^{122,124} \mathrm{Cd}$ coincide with beyond-mean-field calculations with a resultant prolate deformation, whereas ${ }^{126,128} \mathrm{Cd}$ are better described by shell-model calculations.
\end{abstract}

\section{Introduction}

The neutron-rich cadmium nuclei with a proton number of $\mathrm{Z}=48$ are some of the most interesting isotopes in nuclear structure physics. Due to the proximity to the proton and neutron shell-closures at $\mathrm{Z}=50$ and $\mathrm{N}=82$, respectively, a regular behaviour of nuclear quantities is expected. The excitation energies of the $2_{1}^{+}$-states in isotopes close to the neutron-rich Cd isotopes increase towards the $\mathrm{N}=82$ shell-closure. Figure 1 reveals the unexpected behaviour of the $\mathrm{Cd}$ isotopes. In the progression from

\footnotetext{
ae-mail: sboenig@ikp.tu-darmstadt.de
}

This is an Open Access article distributed under the terms of the Creative Commons Attribution License 2.0, which permits unrestricted use, distribution, and reproduction in any medium, provided the original work is properly cited. 
${ }^{122} \mathrm{Cd}$ to ${ }^{126} \mathrm{Cd}$, only a slight increase in excitation energy can be noted, which evolves even to a decrease for ${ }^{126} \mathrm{Cd}$ to ${ }^{128} \mathrm{Cd}$. Although the shell-closure is near, this finding can not be reproduced by shell-model (SM) calculations, yet. Only beyond mean field (BMF) calculations with a resultant prolate deformation agree with the low excitation energy of ${ }^{124-128} \mathrm{Cd}[2]$.

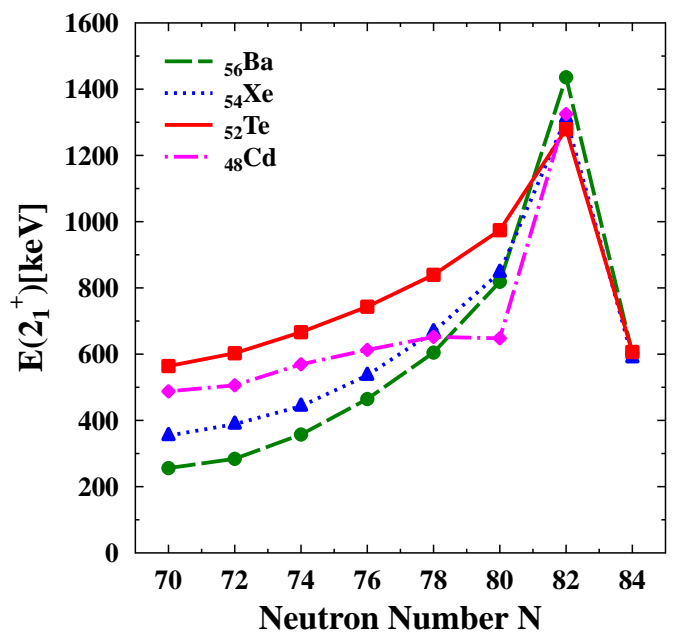

Figure 1. Excitation energies of the $2_{1}^{+}$states for different isotopes [1]. Note the untypical behaviour of the $\mathrm{Cd}$ isotopes.

An important quantity in nuclear structure physics is the transition strength, which gives information about the collectivity of a nucleus. For the investigated $\mathrm{Cd}$ nuclei different predictions exist: $\mathrm{BMF}$ calculations give a rather high $\mathrm{B}\left(\mathrm{E} 2,0_{\mathrm{gs}}^{+} \rightarrow 2_{1}^{+}\right)$value, $\mathrm{SM}$ calculations foresee a much lower strength. In order to experimentally investigate the evolution of the transition strength in this region, a Coulomb excitation experiment was performed. As these nuclei are highly exotic, it is very difficult to produce a beam with a sufficiently high yield to approach the above mentioned quantity. However, at REX-ISOLDE (CERN) it became possible to deliver beams of these exotic nuclei such that with the use of the MINIBALL detector array the different theoretical approaches can be tested in this special region.

\section{The Experiment}

The following description of the performed experiment will focus on the special case of ${ }^{128} \mathrm{Cd}$. Note that the Coulomb excitation experiments on ${ }^{122-126} \mathrm{Cd}$ have been performed in a similar way.

\subsection{Beam and Setup}

The neutron-rich Cd nuclei were produced by $1.4 \mathrm{GeV}$ proton bunches impinging on a primary $\mathrm{UC}_{\mathrm{x}}$ target. The fission products diffuse through the heated target and are extracted into the transfer line. In order to purify the beam, a resonant ionisation laser ion source (RILIS [3]) was used to selectively ionise the $\mathrm{Cd}$ atoms. After the extraction the ions were mass separated by $\mathrm{A} / \mathrm{q}$ with the high resolution 
separator (HRS). In the REX-ISOLDE post accelerator [4] the ions were cooled, bunched, charge bred and once again mass separated $(\mathrm{A} / \mathrm{q} \simeq 4.267)$. The beam was further accelerated up to $2.82 \mathrm{MeV} / \mathrm{u}$ and hit the secondary target $\left({ }^{64} \mathrm{Zn}, 1.48 \mathrm{mg} / \mathrm{cm}^{2}\right)$ with an averaged total beam intensity of roughly $3 \cdot 10^{3}$ ions/s. When impinging on the secondary target, the projectile and target nuclei undergo Coulomb excitation, which is the excitation of nuclei due to electromagnetic scattering. Because of the low beam energy ( $\simeq 68 \%$ of the Coulomb barrier), no nuclear reactions took place and the process can be called "safe". With the MINIBALL array, which consists of 24 segmented high-purity germanium detectors, the deexcitation $\gamma$ rays were detected. The detection of the scattered particles with a segmented double-sided silicon-strip detector (DSSSD) allows for the determination of the scattering angles and Doppler correction [5].

\subsection{Contamination and final $\gamma$ spectrum}

The beam impinging on the secondary ${ }^{64} \mathrm{Zn}$ target was not pure. The $\Delta E-E$ telescope, consisting of an ionisation chamber and a silicon detector situated behind the secondary target, reveals a huge contamination of ${ }^{128} \mathrm{Cs}$. Due to problems with the primary target, the beam composition was not stable over time. Because of this and the fact, that the secondary target has to be taken out of the beam every time a measurement with the telescope takes place, an accurate determination of the beam purity via the telescope is not possible. Fortunately an advantage can be taken of the different half-lifes of ${ }^{128} \mathrm{Cd}\left(\mathrm{T}_{1 / 2}=430 \mathrm{~ms}\right)$ and ${ }^{128} \mathrm{Cs}\left(\mathrm{T}_{1 / 2}=3.6 \mathrm{~min}\right)$. As the protons hit the $\mathrm{UC}_{x}$ target in pulses, the time difference between two consecutive pulses can be used. Setting a gate on short and on large time differences between proton impact and event in the MINIBALL detector, two different $\gamma$ spectra are obtained. The spectrum for large time differences shows no evidence of ${ }^{128} \mathrm{Cd}$, as all the nuclei have already decayed. By normalising the spectra to the deexcitation peaks of ${ }^{128} \mathrm{Cs}$, they can be subtracted from each other and the contamination of ${ }^{128} \mathrm{Cs}$ as well as any other long-lived beam component is eliminated.

In order to determine the short-lived contamination, a thick target of ${ }^{64} \mathrm{Zn}\left(14.89 \mathrm{mg} / \mathrm{cm}^{2}\right)$ was used. As the beam was implanted in the target, the beam composition can be determined via characteristic $\beta$-decay lines. Besides the ${ }^{128} \mathrm{Cs}$, also a contamination of ${ }^{128} \mathrm{In}\left(\mathrm{T}_{1 / 2}=840 \mathrm{~ms}\right)$ and ${ }^{128 \mathrm{~m}} \mathrm{In}$ $\left(\mathrm{T}_{1 / 2}=720 \mathrm{~ms}\right)$ could be deduced. The beam composition without taking the ${ }^{128} \mathrm{Cs}$ into account is roughly given as: $\sim 55 \%{ }^{128} \mathrm{Cd}, \sim 40 \%{ }^{128} \mathrm{In}$ and $\sim 5 \%{ }^{128 \mathrm{~m}} \mathrm{In}$.

Figure 2 shows the subtracted and Doppler corrected $\gamma$ spectrum. Clearly visible are the $\mathrm{Zn}$ and the Cd excitation peaks. The unknown line at $324 \mathrm{keV}$ can be assigned to a new transition in the contaminating nucleus ${ }^{128} \mathrm{In}$.

\section{Results}

From the obtained $\gamma$ spectrum, a Coulomb excitation cross-section for the process of interest can be deduced in a semi-classical approach. The $\mathrm{B}(\mathrm{E} 2)$-value is a function of the transitional and diagonal matrix elements of the involved nuclear states (here these are $\left\langle 2_{1}^{+}\|\mathrm{M}(\mathrm{E} 2)\| 0_{g s}^{+}\right\rangle$and $\left\langle 2_{1}^{+}\|\mathrm{M}(\mathrm{E} 2)\| 2_{1}^{+}\right\rangle$). A variation of those matrix elements until an agreement between calculated and experimentally found cross-section is achieved leads to the extraction of a transition strength. The values are obtained relative to the target excitation to reduce effects such as from detector efficiencies.

Preliminarily it can be said, that for ${ }^{122,124} \mathrm{Cd}$ the transition strength agrees with the rather large values of BMF calculations [6]. The transition strength of ${ }^{126,128} \mathrm{Cd}$ seems to be described well by SM calculations, as one would expect due to the proximity to the closed proton and neutron shells $[6,7]$. Nevertheless, it is still an open question why the drop in excitation energy can not be reproduced by the theoretical approach that describes the $\mathrm{B}\left(\mathrm{E} 2,0_{\mathrm{gs}}^{+} \rightarrow 2_{1}^{+}\right)$value and vice versa. 


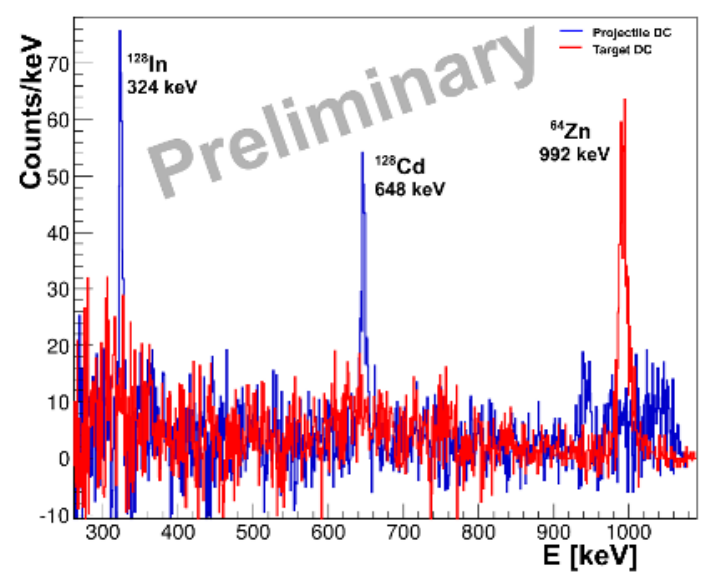

Figure 2. $\gamma$ spectrum with indicated transitions in projectile and target nuclei (time gated, subtracted, background subtracted and Doppler corrected), full statistics. A new transition in the ${ }^{128}$ In contaminant can be seen.

\section{Outlook}

Recently, a first Coulomb excitation experiment on odd Cd isotopes was performed at REX-ISOLDE. The campaign started with the investigation of ${ }^{123} \mathrm{Cd}[8]$. By populating the ground and the isomeric state and extracting the transition strengths connected to these states, it will be possible to assign the collectivity to particular orbits. The campaign on collectivity in neutron-rich $\mathrm{Cd}$ isotopes will be continued at HIE-ISOLDE, which is now under construction and will deliver more energetic and intense radioactive ion beams than ISOLDE.

\section{Acknowledgements}

This project is supported by BMBF (No. 06 DA 9036I, No. 05 P12 RDCIA, No. 05 P12 RDCIB and No. 05 P12 PKFNE), HIC for FAIR, EU through EURONS (No. 506065) and ENSAR (No. 262010) and the MINIBALL and REX-ISOLDE collaborations.

\section{References}

[1] http://www.nndc.bnl.gov/ (July 2013)

[2] T. Rodriguez, J. Egido, A. Jungclaus, Phys. Lett. B 668, 410-413 (2008)

[3] V.N. Fedosseev et al., Nucl. Instrum. Methods Phys. Res. B 266, 4378-4382 (2008)

[4] O. Kester et al., Nucl. Instr. and Meth. B 204, 20-30 (2003).

[5] N. Warr et al., Eur. Phys. J. A 49, 40 (2013)

[6] S. Ilieva et al., to be published

[7] S. Bönig et al., to be published

[8] T. Kröll et al., CERN Proposal INTC-P-306 (2011) 